\title{
Guiding the swing in golf putting
}

\section{Golfers control the pace of a putt by comparing sensory data with an internal guide.}

A ctions that involve making contact with surfaces often demand perceptual regulation of the impact — for example, of feet with ground when walking or of bat with ball when hitting. Here we investigate how this control of impact is achieved in golf putting, where control of the clubhead motion at ball impact is paramount in ensuring that the ball will travel the required distance. Our results from ten professional golfers indicate that the clubhead motion is spatially scaled and perceptually regulated by coupling it onto an intrinsic guide generated in the nervous system.

Our model of motor control in putting considers two fundamental but largely ignored issues ${ }^{1,2}$ : the role that information gathered through the senses plays in guiding actions, and the control processes used by the nervous system to solve the guidance problem. This model (Box 1, overleaf) is based on a general theory ${ }^{3-5}$ that any guiding movement must involve coordinating and regulating the rate of closure of motion gaps, such as the gap between a club and ball.

The theory argues that gap closure is controlled by using perceptual information about a particular measure of a gap, denoted as $\tau$, which is equal to the time to gap closure at the existing closure rate. The rate of gap closure can be regulated by keeping the $\tau$ value of the motion gap coupled onto (in constant ratio with) the $\tau$ value of a 'guiding gap'. In self-paced actions like putting, this $\tau$ guide is assumed to be generated in the nervous system (for example, by modulating energy levels). Neural mechanisms are implicated in the intrinsic timing of movements ${ }^{6-8}$ and in their spatial specification $^{9}$, and there are similarities in brain activity when the performance of an action is being imagined and when it is subsequently executed ${ }^{10-12}$.

The golfer controls the motion pattern of the forward swing of the putting action by constantly sensing the $\tau$ of the gap between the club and the end of the follow-through, and keeping this $\tau$ in constant ratio with an intrinsic $\tau$ guide (Box 1, Fig. 1a) ${ }^{4,5}$. Spatially scaling the forward-swing pattern generated by the intrinsic $\tau$ guide could be achieved by adjusting one or more of four possible parameters (Box 1): the amplitude, $D$, of the forward swing (more specifically, $D^{2}$ ); the duration, $T$, of the forward swing (more specifically, $\left.1 / T^{2}\right)$; the relative time, $P_{T}$, at which the ball is hit; and the $\tau$-coupling constant, $k$. The first two parameters are the most plausible as they alone are linearly related to the distance the ball will travel (equation (2) in Box 1).
To test this model, we analysed the putting actions of ten low-handicap (under 5) golfers across different distances. The motion pattern was $\tau$-guided, as evidenced by the strong linear relation between the temporal evolution of the $\tau$ of the forward swing and the intrinsic $\tau$ guide (Fig. 1b). The proportion of energy loss at club-ball impact and the retarding force on the ball were both relatively constant, as assumed by the model, with $r^{2}$ linear-regression values of the club's impact velocity (squared) onto
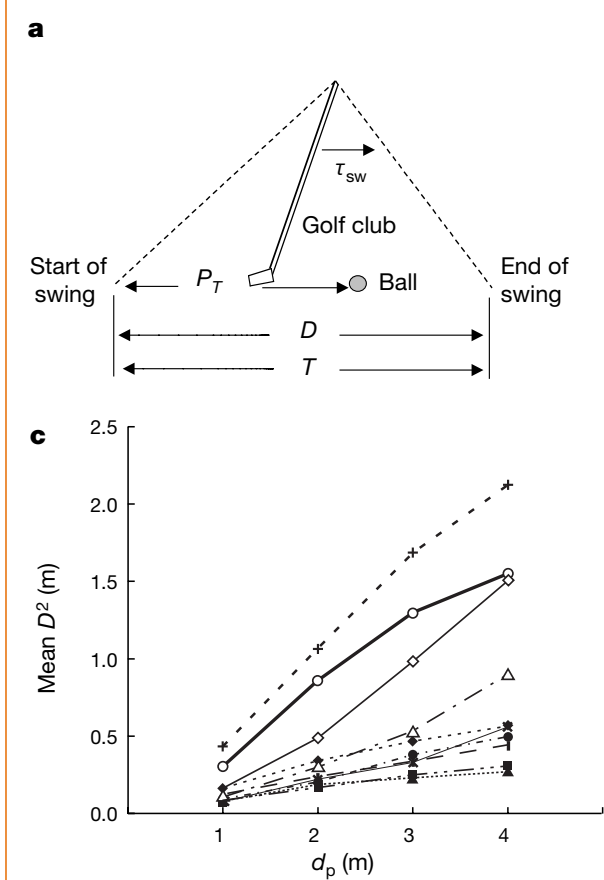

putting distance being in the range 0.985 to 0.999. Spatial scaling was brought about by adjusting $D^{2}$, whereas the other three parameters showed less systematic change with putting distance (Fig. 1c-f).

Our model predicts how golfers regulate the spatial and temporal components of the forward swing in order to transmit the appropriate amount of kinetic energy at ball impact. The same principles - of guiding movements by coupling them onto intrinsic $\tau$ guides and spatially scaling the
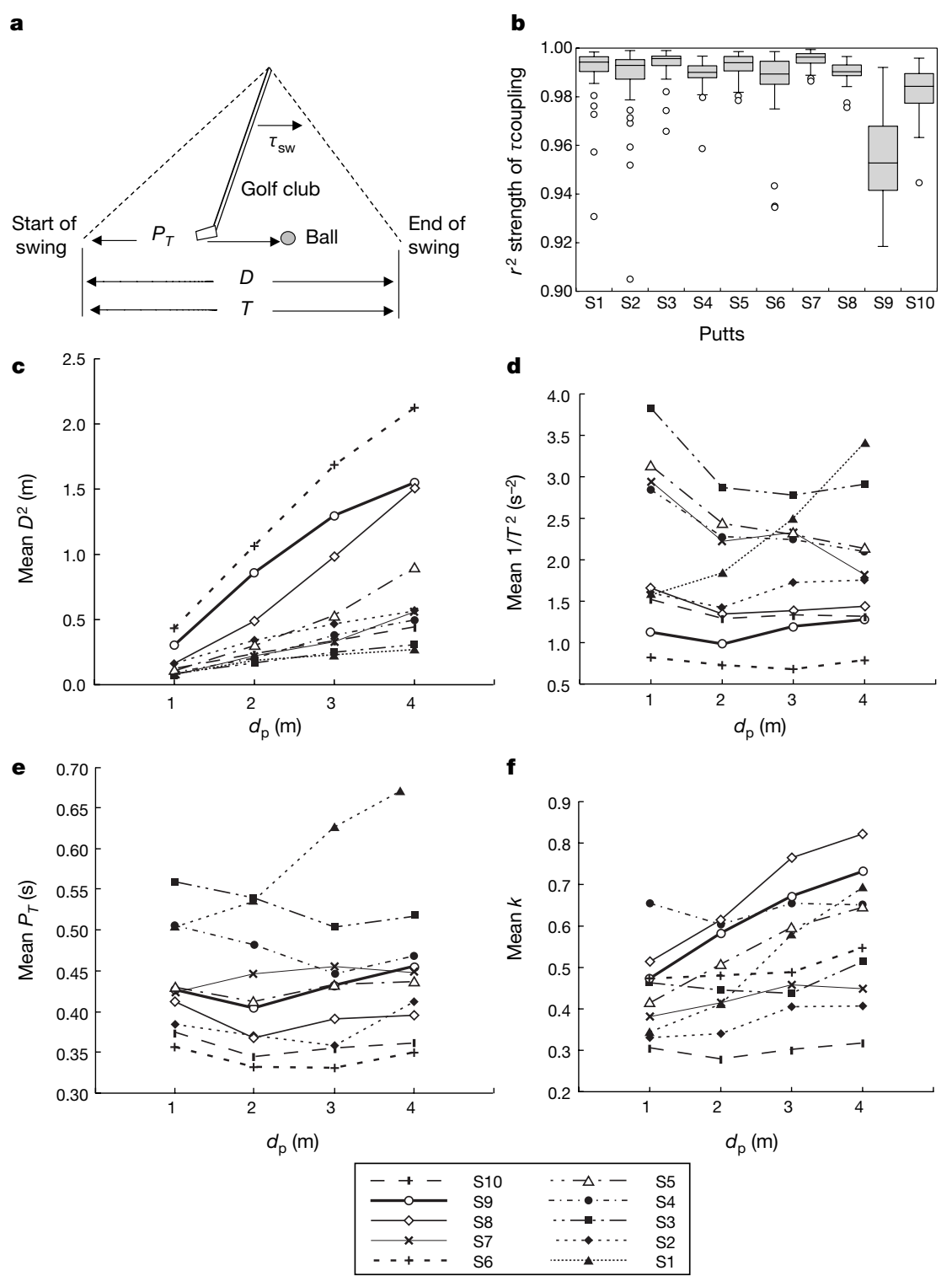

Figure $1 \tau$-guiding the forward swing. Ten golfers' club movements were recorded at $200 \mathrm{~Hz}$ when putting to four distances, ten times, on an artificial green. a, Motion parameters defined in Box 1. Horizontal distance/velocity ratio for the clubhead during the forward swing was a measure of the $\tau_{\mathrm{sw}}$. For each putt, $\tau_{\mathrm{Sw}}$ was linearly regressed onto the intrinsic $\tau$ guide $\left(\tau_{\mathrm{g}}\right)$ to determine the degree of linearity $\left(r^{2}\right)$. b, Range of the proportions of variance accounted for by the $\tau$-coupling model (as measured by $r^{2}$ values) for golfers S1-S10. c-f, Relation between the parameters $\left(D^{2}, 1 / T^{2}, P_{T}\right.$ and $\left.k\right)$ and putt distance, $d_{p}$, for each of the ten golfers. 


\section{Box 1 The golfer's $\tau$ guide to putting}

The golfer guides the forward swing by coupling $\tau_{\mathrm{sw}}$ (time to gap closure, at the prevailing closure rate of the clubhead with the end of the follow-through) onto an intrinsic $\tau$-guide, $\tau_{\mathrm{g}}=$

$0.5\left(t-T^{2} / t\right)$, where $t$ is the time from the start of the swing and $T$ is its duration. Thus, the relation $\tau_{\mathrm{sw}}=$

$k \tau_{g}=0.5 k\left(t-T^{2} / t\right)$ is maintained during the forward swing ( $k$ is a coupling con- stant). Integration leads to

$V_{c}=2 D(1 / T)\left(P_{T} / k\right)\left(1-P_{T}^{2}\right)^{(1 / k)-1}$

where $V_{c}$ is the clubhead velocity just

before impact, $D$ is the amplitude of the forward swing and $P_{T}$ is the proportion of the swing duration before the ball is hit. The kinetic energy of the club-body system (effective mass, $M_{c}$ ) just before impact is equal to $M_{c} V_{c}^{2} / 2$. Assuming a constant proportion of energy is lost during impact and the ball's motion is opposed by a constant force $F$, equation (2) gives the distance putted $\left(d_{p}\right)$ as $d_{p}=\lambda M_{\mathrm{c}} V_{\mathrm{c}}^{2} / 2 F=\left(2 \lambda M_{\mathrm{c}} / F\right) D^{2}\left(1 / T^{2}\right)\left(P_{T} / k\right)^{2}$ $\left(1-P_{T}^{2}\right)^{(2 / k)-2}$, where $\lambda$ is a constant. Thus, the golfer could scale the distance putted by adjusting $D^{2}, 1 / T^{2}, P_{T}$ or $k$. As $D^{2}$ and $1 / T^{2}$ are each linearly related to distance, these are the more likely candidates. movements - might apply generally when regulating impact force with objects and surfaces. The parameter used in scaling, however, might vary with the task.

C. M. Craig ${ }^{\star}$, D. Delay $\dagger$, M. A. Grealy

D. N. Lee\$

${ }^{*}$ UMR Mouvement and Perception, 163 Avenue de Luminy, Université de la Méditerranée,

13288 Marseilles, France

e-mail: craig@laps.univ-mrs.fr

$\dagger$ UFRAPS, Université de Joseph Fourier,

38041 Grenoble, France

$\ddagger$ Department of Psychology, Strathclyde University,

Graham Hills Building, Glasgow G1 1XQ, UK

\section{Natural selection}

\section{Evolution of lifespan in C. elegans}

$\mathrm{t}$ was proposed almost 50 years ago that ageing is non-adaptive and is the consequence of a decline in the force of natural selection with age ${ }^{1}$. This led to the theory that ageing results from detrimental effects late in life of genes that act beneficially in early life ${ }^{1,2}$, so any genetic alteration that increases lifespan might be expected to reduce fitness, for example. We show here that a mutation that greatly increases the elegans does indeed exhibit a fitness cost, as demonstrated during starvation cycles that may mimic field conditions, thereby validating the pleiotropy theory of ageing ${ }^{2}$.

C. elegans is a soil-dwelling nematode with a facultative, self-fertilizing mode of reproduction. Mutation of the age-1 gene, which encodes a phosphatidylinositol 3-OH kinase catalytic subunit component of the insulin-like signalling pathway ${ }^{3}$, can extend adult lifespan by up to $80 \%$ (ref. 4 ). The age-1 gene, and other genes encoding components of the insulin-like signalling pathway, not only influence ageing, but also control progress of normal development ${ }^{5-7}$ and determine adult stress resistance ${ }^{8}$.

If worms carrying the weak mutant allele age-1( $h \times 546)$ are grown at $27^{\circ} \mathrm{C}$, they develop into dauer larvae (a diapause stage) that does not feed or reproduce ${ }^{5}$, but if they are grown at $20^{\circ} \mathrm{C}$, they develop normally lifespan of the nematode Caenorhabditis
\$Department of Psychology, University of Edinburgh, 7 George Square, Edinburgh EH8 9JZ, UK

1. Kelso, J. A. S. Hum. Mov. Sci. 10, 93-111 (1991).

2. Hudson, J. L. Med. Sci. Sport Ex. 18, 242-251 (1986).

3. Lee, D. N. Ecol. Psychol. 10, 221-250 (1998).

4. Craig, C. M. \& Lee, D. N. Exp. Brain Res. 124, 371-382 (1999).

5. Lee, D. N., Craig, C. M. \& Grealy, M. A. Proc. R. Soc. Lond. B 266, 2029-2035 (1999).

6. Ivry, R. \& Keele, S. J. Cogn. Neurosci. 1, 136-152 (1999).

7. Nichelli, P., Always, D. \& Grafman, J. Neuropsychology 34,

8. Meck, W. H. Cogn. Brain Res. 3, 227-242 (1996).

9. Georgopoulus, A. P. \& Massey, J. T. Exp. Brain Res. 65, 361-370 (1987).

10. Jeannerod, M. Neuropsychology 33, 1419-1432 (1995). 11. Jeannerod, M. Behav. Brain Sci. 17, 187-245 (1999). 12. Georgopoulus, A. P. et al. Science 243, 234-236 (1989).

into adults. At this growth temperature, mutant and wild-type worms are essentially identical in appearance, development rates, activity levels and total fertility ${ }^{4,9,10}$.

Alleles that confer an extended lifespan but appear otherwise normal are at odds with the pleiotropy theory ${ }^{1,2}$. We have tested this theory by measuring the relative fitness of the $h \times 546$ and wild-type alleles of age-1 in strains that are otherwise isogenic.

We established synchronously ageing populations of hermaphrodite worms, each containing wild-type and age-1( $h \times 546)$ worms on the same agar plates at $20^{\circ} \mathrm{C}$. As there were no males in these populations, all

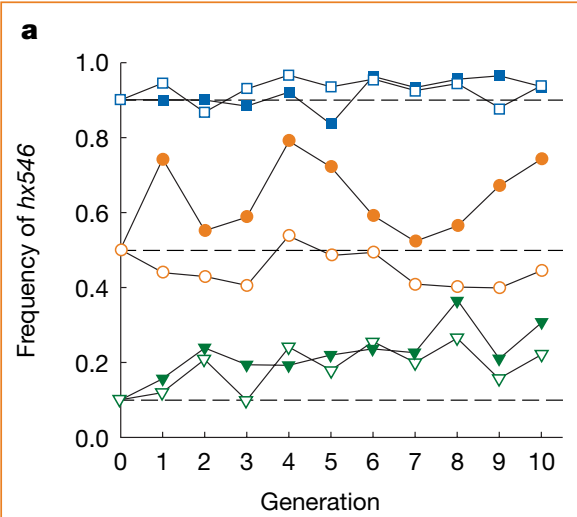
863-871 (1996).

Figure 1 Direct competition between long-lived and wild-type worms in laboratory natural-selection experiments. a, Frequencies of age-1(hx546) under non-starvation conditions at $20^{\circ} \mathrm{C}$ in mixed populations of N2 (wild type) and TJ1052 (age-1(hx546)) worms. b, Frequencies of age-1(hx546) in five replicate populations cultured with cyclical starvation, where the worms were depleted of all food by five days and remained starved for a further four days. Each starvation cycle approximated two generations. For a reduction in allele frequency of 0.44 in 12 generations, the relative fitness of age-1(hx546) versus wild type is estimated to be 0.77 , indicating a major cost to age-1(hx546) under these conditions. 